\title{
Therapeutic role of corticosteroids in COVID-19: a systematic review of registered clinical trials
}

\author{
Reshma Raju, Prajith V., Pratheeksha Sojan Biatris and Sam Johnson Udaya Chander J."
}

\begin{abstract}
Background: In March 2020, the World Health Organization declared the coronavirus disease 2019 as a global pandemic. Though antiviral drugs and antimalarial drugs are considered treatment options for treating coronavirus disease 2019 (COVID-19), no specific antivirals are currently available for its treatment. Efficient use of drug discovery approaches including repurposing or repositioning of drugs used in the treatment of severe acute respiratory syndrome coronavirus (SARS-CoV) and the Middle East respiratory syndrome coronavirus (MERS-CoV) is considered recently. The widespread application of corticosteroid therapy in COVID-19 should be backed with careful documented pragmatic research of its use in this context.

Main body: This article aims to analyze various trials registered across the globe providing an overall picture of the use of corticosteroids in the treatment of COVID-19. An extensive search was conducted on the clinical trial registries around the world to identify all the trials reporting information regarding the use of corticosteroids in COVID-19. Our initial search returned 231 trials, out of which 60 trials were finally included in the analysis. Fifty-six studies were interventional trials, and all the trials had clearly defined primary and secondary outcomes of interest, of which only 11 trials had evaluation of respiratory rate as one of their outcomes.
\end{abstract}

Conclusion: Few preliminary trial findings show promising results and recommend the use of methylprednisolone and dexamethasone in the severe form of the disease; however, there is insufficient data to prove its benefits over its risks. Routine use of corticosteroids should be favored only after a better insight is obtained, with the completion of these trials.

Keywords: Methylprednisolone, Hydrocortisone, Steroids, COVID-19, SARS-CoV2, Clinical trial registry

\section{Background}

An outbreak of pneumonia caused by a new coronavirus spread in Wuhan province of China in December 2019. Sequencing of the sampling from patients with pneumonia revealed the viral genome phylogenetically closer to severe acute respiratory syndrome coronavirus (SARS$\mathrm{CoV})$ and the Middle East respiratory syndrome coronavirus (MERS-CoV) [1]. The Coronavirus Study Group named the causative agent severe acute respiratory syndrome coronavirus 2 (SARS-CoV-2), and the disease caused by this virus was named coronavirus disease 2019 (COVID-19 or 2019-nCoV) by the World Health

\footnotetext{
* Correspondence: mail2samjohnson@gmail.com

College of Pharmacy, Sri Ramakrishna Institute of Paramedical Sciences, Coimbatore, Tamil Nadu, India
}

\section{Springer Open}

Organization (WHO) [2, 3]. These viruses are enveloped, positive, single-stranded RNA viruses belonging to the family Coronaviridae, which can cause an array of symptoms including fever, dry cough, myalgia, fatigue, and dyspnea [4]. SARS-CoV-2 transmits from human-tohuman by respiratory droplets caused by coughing or sneezing $[5,6]$. The WHO declared COVID-19 as a Public Health Emergency of International Concern in January 2020 [7]. The infection has spread over to 216 countries $(15,745,102$ confirmed cases and 639,317 confirmed deaths) since its outbreak in November 2019 (as of 30 January 2021; Fig. 1).

Detection and diagnosis of this novel coronavirus mostly relied on molecular-based approaches such as nucleic acid testing, virus antigen, or serological
(ㅇ The Author(s). 2021 Open Access This article is licensed under a Creative Commons Attribution 4.0 International License, which permits use, sharing, adaptation, distribution and reproduction in any medium or format, as long as you give appropriate credit to the original author(s) and the source, provide a link to the Creative Commons licence, and indicate if changes were made. The images or other third party material in this article are included in the article's Creative Commons licence, unless indicated otherwise in a credit line to the material. If material is not included in the article's Creative Commons licence and your intended use is not permitted by statutory regulation or exceeds the permitted use, you will need to obtain permission directly from the copyright holder. To view a copy of this licence, visit http://creativecommons.org/licenses/by/4.0/. 


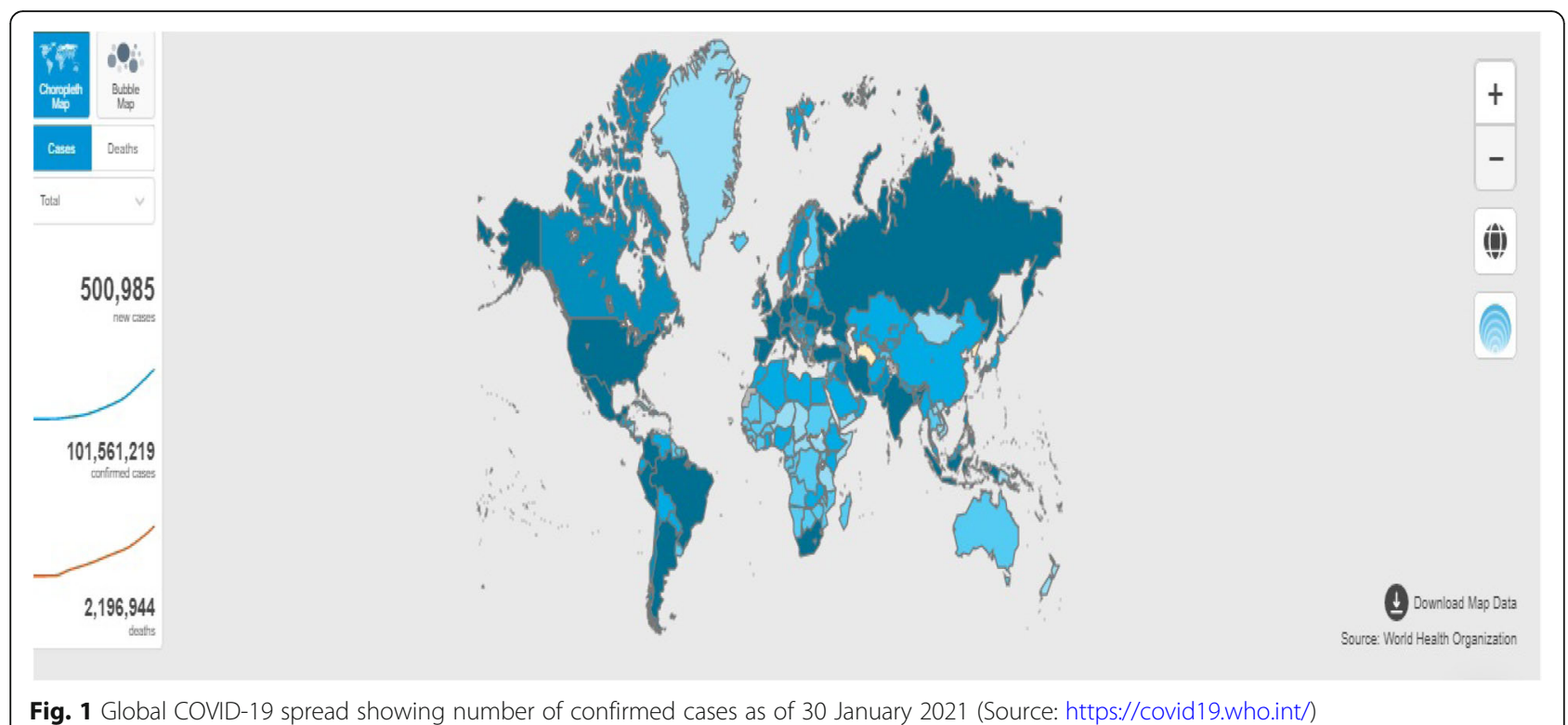

Fig. 1 Global COVID-19 spread showing number of confirmed cases as of 30 January 2021 (Source: https://covid19.who.int/)

antibody testing (against the N-protein of SARS-CoV) [8]. Treatment option includes antiviral drugs such as favipiravir, remdesivir, lopinavir, and ritonavir and antimalarial drugs such as chloroquine or hydroxychloroquine. Nevertheless, no vaccine or specific antiviral treatment recommended for COVID-19 is currently available [9]. The uncontrolled scenario of COVID-19 demands the use of effective drug discovery approaches for effective control of the disease [10-16]. Among these approaches, drug repurposing or drug repositioning is a time-effective way of treating a disease. One of the examples of successful application of drug discovery approach is drug repositioning of antivirals, and it has triggered a number of in vitro studies as well as clinical trials for a number of chemical molecules to evaluate their efficacy against COVID-19 [17-19]. Drug repurposing of corticosteroids has also been implemented recently as a part of a drug discovery approach. There are several studies reporting the use of corticosteroids in the treatment of severe coronavirus infections including COVID-19. The effectiveness of corticosteroids in some patients with SARS-CoV has resulted in a widespread application of this therapy in COVID-19, especially in patients in the ICU with severe infections, as these drugs prevent lung injury caused by severe communityacquired pneumonia (sCAP) due to their potential pharmacological effects on the suppression of exuberant and dysfunctional systematic inflammation [20].

\section{Main body}

\section{Corticosteroids and their therapeutic role}

The Infectious Diseases Society of America (IDSA) guidelines strongly recommends the use of dexamethasone in critically ill patients to treat acute respiratory distress syndrome (ARDS) and systemic inflammation, backed by moderate evidence. Dexamethasone at a total daily dose of $6 \mathrm{mg}$ IV or PO for 10 days (or until discharge) or alternative glucocorticoids like methylprednisolone $32 \mathrm{mg}$ and prednisone $40 \mathrm{mg}$ are suggested. The level of recommendation decreases with decreasing severity of the disease. In non-severe COVID-19, the use of glucocorticoids is not recommended as there is a dearth of solid evidence. Additionally, experiences from SARS and MERS show risk of worsening clinical status, delayed viral clearance, and other adverse events [21]. Currently, available data on safety and effectiveness of corticosteroids in this setting is very few and inconclusive [20,22, 23]. The value of corticosteroids as a treatment option in patients with severe COVID-19 infection needs careful documented pragmatic research in this context. In order to obtain strong clinical evidence, several studies have been launched that were registered on various clinical trial registries across the globe. The detailed analysis of these trials will give an overall picture of the use of corticosteroids in the treatment of COVID-19 around the world. This will help to identify the lacunae to be filled with definitive clinical evidence in order to reposition corticosteroid for COVID-19 treatment. Therefore, this study aims to analyze various trials registered across the globe providing an overall picture of the use of corticosteroids in the treatment of COVID-19.

\section{Search strategy}

An extensive search was conducted to identify all the trials reporting information regarding the use of corticosteroids in COVID-19. We searched the following clinical 
trial registries: Clinicaltrials.gov, Chinese Clinical Trial Registry (ChiCTR), Clinical Research Information Service (CRiS)-Republic of Korea, EU Clinical Trials Register, ISRCTN Registry, Iranian Registry of Clinical Trials (IRCT), German Clinical Trials Register (DRKS), Japan Primary Registries Network (JPRN), and Clinical Trial Registry-India. The search was run until 23 June 2020. In Clinicaltrials.gov, the following keywords were used for search: "(COVID-19 OR SARS-CoV-2 OR 2019nCoV OR severe acute respiratory syndrome coronavirus 2 OR Wuhan coronavirus OR 2019 novel coronavirus OR novel coronavirus-infected Pneumonia) AND ("glucocorticoids" OR "steroids" OR "corticosteroids" OR "hydrocortisone" OR "prednisone" OR "methylprednisolone" OR "dexamethasone" OR "prednisolone"). A similar strategy was adapted for the other registries. We included the English language and interventional and non-interventional studies. No restrictions were placed on the dose or formulation of the intervention. All trials must have studied the safety and efficacy of steroids in COVID-19 care.

\section{Recovery of trials}

Our initial search returned 231 trials, out of which 62 potentially relevant trials were identified. Potentially eligible trials were identified by three authors by screening titles and study description. All eligible trials were then assessed independently by three authors, and potentially relevant trials were selected in accordance with the predefined inclusion criteria. Any disagreement was reviewed and resolved by a fourth independent reviewer. Authors of individual trials were contacted if necessary. After a careful review of the study description, out of 62 articles, 2 trials did not satisfy the inclusion criteria and were excluded from the analysis. Finally, data from 60 trials were included in the final review and synthesis of results. This is shown in Fig. 2.

\section{Data abstraction and study appraisal}

We extracted the following general data from each study: trial number, title, origin (country) of study, intervention, treatment arms, doses, mean age of participants, stage of COVID-19, expected start and end date of trial,

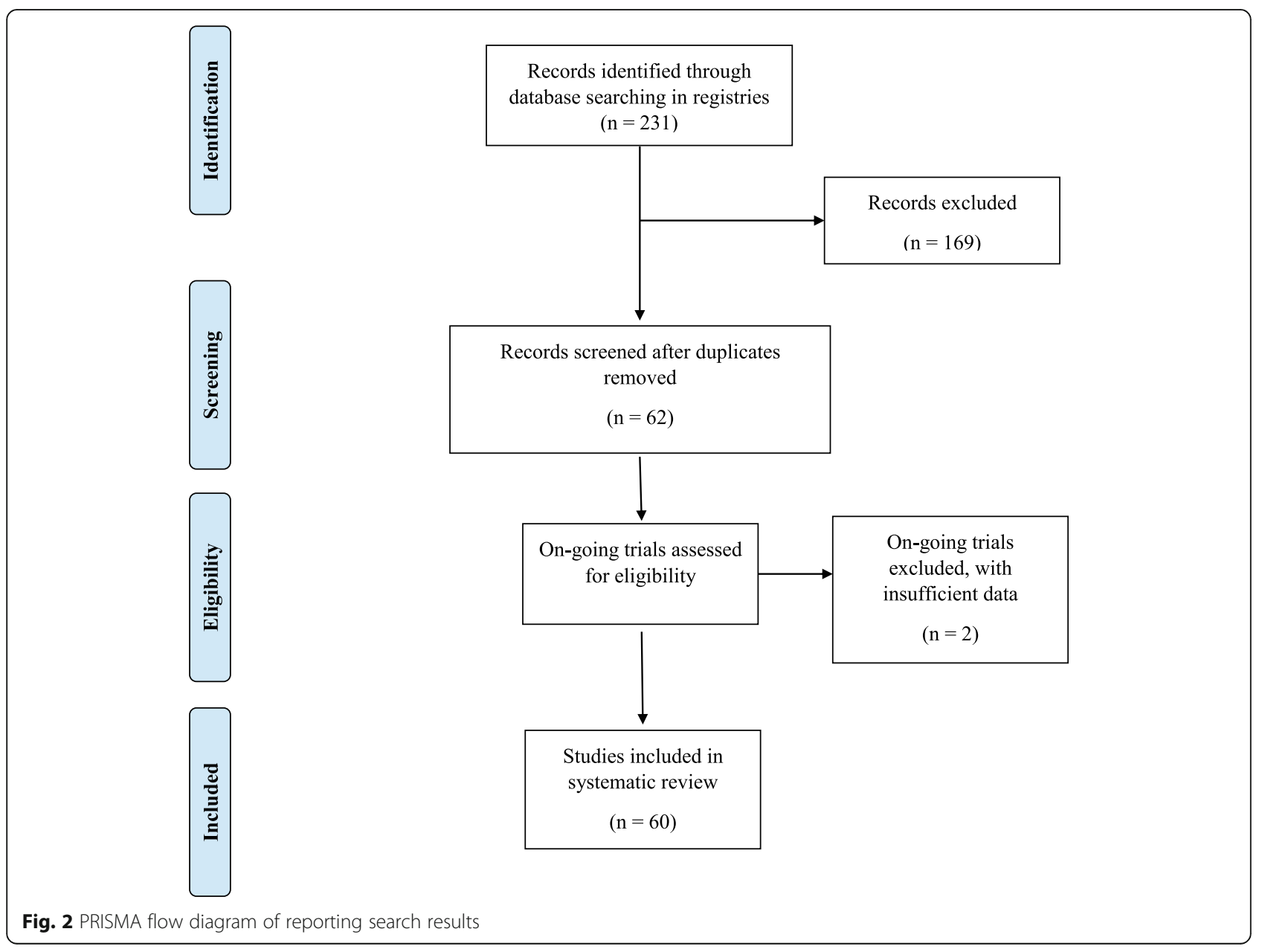


Table 1 General characteristics of the included trials

\begin{tabular}{|c|c|c|c|c|c|c|}
\hline Trial identifier & Country & $\begin{array}{l}\text { Number } \\
\text { of sites }\end{array}$ & Start date & $\begin{array}{l}\text { Expected } \\
\text { completion } \\
\text { date }\end{array}$ & $\begin{array}{l}\text { Stage of } \\
\text { COVID-19 }\end{array}$ & Outcome measures \\
\hline NCT04425863 & Argentina & Single & May 2020 & July 2020 & $\begin{array}{l}\text { Severe acute } \\
\text { respiratory } \\
\text { syndrome }\end{array}$ & $\begin{array}{l}\text { 1. Illness development } \\
\text { 2. Reduction of ICU admission } \\
\text { 3. Mortality rate }\end{array}$ \\
\hline NCT04395105 & Argentina & Multi & May 2020 & $\begin{array}{l}\text { January } \\
2021\end{array}$ & $\begin{array}{l}\text { Respiratory } \\
\text { distress } \\
\text { syndrome }\end{array}$ & Ventilator-free days at 28 days \\
\hline NCT02735707 & $\begin{array}{l}\text { Australia, Belgium, Canada, } \\
\text { Croatia, Germany, Hungary, } \\
\text { Ireland, Netherlands, New } \\
\text { Zealand, Portugal, Romania, } \\
\text { Spain, UK }\end{array}$ & Multi & April 2020 & $\begin{array}{l}\text { December } \\
2023\end{array}$ & Pneumonia & $\begin{array}{l}\text { 1. All-cause mortality } \\
\text { 2. Days alive and outside of ICU }\end{array}$ \\
\hline NCT04343729 & Brazil & Single & April 2020 & $\begin{array}{l}\text { September } \\
2020\end{array}$ & $\begin{array}{l}\text { Severe acute } \\
\text { respiratory } \\
\text { syndrome } \\
\text { (SARS) }\end{array}$ & Mortality rate \\
\hline NCT04327401 & Brazil & Multi & April 2020 & August 2020 & $\begin{array}{l}\text { Moderate/ } \\
\text { severe ARDS }\end{array}$ & Ventilator-free days \\
\hline NCT04377503 & Brazil & $\begin{array}{l}\text { Not } \\
\text { available }\end{array}$ & May 2020 & $\begin{array}{l}\text { November } \\
2020\end{array}$ & $\begin{array}{l}\text { Cytokine } \\
\text { release } \\
\text { syndrome }\end{array}$ & $\begin{array}{l}\text { Patient clinical status } 15 \text { days after } \\
\text { randomization }\end{array}$ \\
\hline NCT04374474 & Canada & Single & $\begin{array}{l}\text { January } \\
2021\end{array}$ & March 2022 & Not defined & $\begin{array}{l}\text { 1. Change from Baseline Snap and } \\
\text { Sniff Threshold Test at } 3 \text { months } \\
\text { 2. Score from the Snap and Sniff } \\
\text { Olfactory Test results } \\
\text { 3. Change from baseline Smell } \\
\text { Identification Test (SIT) at } 3 \\
\text { months } \\
\text { 4. Score from the Smell } \\
\text { Identification test results. } \\
\text { 5. Change from Baseline Snap and } \\
\text { Sniff Threshold Test at } 6 \text { months } \\
\text { 6. Score from the Snap and Sniff } \\
\text { Olfactory Test results } \\
\text { 7. Change from baseline Smell } \\
\text { Identification Test (SIT) at } 6 \\
\text { months }\end{array}$ \\
\hline NCT04263402 & China & $\begin{array}{l}\text { Not } \\
\text { available }\end{array}$ & $\begin{array}{l}\text { February } \\
2020\end{array}$ & July 2020 & $\begin{array}{l}\text { Severe } \\
\text { pneumonia }\end{array}$ & $\begin{array}{l}\text { 1. Rate of disease remission } \\
\text { 2. Rate and time of entering the } \\
\text { critical stage }\end{array}$ \\
\hline NCT04244591 & China & Completed & $\begin{array}{l}\text { January } \\
2020\end{array}$ & April 2020 & $\begin{array}{l}\text { Severe acute } \\
\text { respiratory } \\
\text { failure }\end{array}$ & Murray lung injury score \\
\hline NCT04273321 & China & Completed & $\begin{array}{l}\text { February } \\
2020\end{array}$ & April 2020 & Pneumonia & $\begin{array}{l}\text { The incidence of treatment failure } \\
\text { in } 14 \text { days }\end{array}$ \\
\hline ChiCTR2000029386 & China & Single & $\begin{array}{l}\text { January } \\
2020\end{array}$ & $\begin{array}{l}\text { January } \\
2021\end{array}$ & Pneumonia & SOFA score \\
\hline ChiCTR2000029656 & China & Single & $\begin{array}{l}\text { February } \\
2020\end{array}$ & April 2020 & Pneumonia & $\begin{array}{l}\text { ECG, chest imaging, complications, } \\
\text { vital signs, and NEWS2 score }\end{array}$ \\
\hline ChiCTR2000030481 & China & Multi & $\begin{array}{l}\text { January } \\
2020\end{array}$ & April 2020 & Pneumonia & $\begin{array}{l}\text { The time of duration of COVID-19 } \\
\text { nucleic acid RT-PCR test results of } \\
\text { respiratory specimens (such as } \\
\text { throat swabs) or blood specimens } \\
\text { change to negative }\end{array}$ \\
\hline NCT04348305 & Denmark & Multi & April 2020 & $\begin{array}{l}\text { December } \\
2021\end{array}$ & $\begin{array}{l}\text { COVID-19 } \\
\text { hypoxia }\end{array}$ & $\begin{array}{l}\text { Days alive without life support at } \\
\text { day } 28\end{array}$ \\
\hline 2020-001395-15 & Denmark & Multi & April 2020 & Not available & $\begin{array}{l}\text { Severe } \\
\text { hypoxia }\end{array}$ & $\begin{array}{l}\text { 1. Days alive without life support } \\
\text { (i.e., invasive mechanical } \\
\text { ventilation, circulatory support or }\end{array}$ \\
\hline
\end{tabular}


Table 1 General characteristics of the included trials (Continued)

\begin{tabular}{|c|c|c|c|c|c|c|}
\hline Trial identifier & Country & $\begin{array}{l}\text { Number } \\
\text { of sites }\end{array}$ & Start date & $\begin{array}{l}\text { Expected } \\
\text { completion } \\
\text { date }\end{array}$ & $\begin{array}{l}\text { Stage of } \\
\text { COVID-19 }\end{array}$ & Outcome measures \\
\hline & & & & & & $\begin{array}{l}\text { renal replacement therapy) from } \\
\text { randomization to day } 28) \text {. }\end{array}$ \\
\hline NCT04331054 & France & Multi & April 2020 & July 2020 & $\begin{array}{l}\text { COVID-19 } \\
\text { infection }\end{array}$ & $\begin{array}{l}\text { Time (in days) to clinical } \\
\text { improvement within } 30 \text { days after } \\
\text { randomization }\end{array}$ \\
\hline NCT04361474 & France & Multi & May 2020 & May 2021 & Hyposmia & $\begin{array}{l}\text { Patient with more than } 2 \text { points } \\
\text { on the ODORATEST }\end{array}$ \\
\hline NCT04359511 & France & $\begin{array}{l}\text { Not } \\
\text { available }\end{array}$ & June 2020 & $\begin{array}{l}\text { December } \\
2020\end{array}$ & Pneumonia & $\begin{array}{l}\text { Clinical improvement defined by } \\
\text { the improvement of } 2 \text { points on a } \\
\text { 7-category ordinal scale, at } 14 \text { days }\end{array}$ \\
\hline NCT04347980 & France & Multi & April 2020 & August 2020 & $\begin{array}{l}\text { Acute } \\
\text { respiratory } \\
\text { distress } \\
\text { syndrome } \\
\text { (ARDS) }\end{array}$ & 28-day mortality \\
\hline NCT04344730 & France & Single & April 2020 & $\begin{array}{l}\text { December } \\
2020\end{array}$ & Pneumonia & $\begin{array}{l}\text { 1. Time-to-death from all causes } \\
\text { within the first } 60 \text { days after } \\
\text { randomization } \\
2 . \text { Time to need for mechanical } \\
\text { ventilation }\end{array}$ \\
\hline NCT04344288 & France & Multi & April 2020 & $\begin{array}{l}\text { November } \\
2020\end{array}$ & Pneumonia & $\begin{array}{l}\text { Respiratory indication for transfer } \\
\text { to intensive care unit evaluated by } \\
\text { a SpO2 }<90 \%\end{array}$ \\
\hline NCT04331470 & Iran & Single & April 2020 & May 2020 & Not defined & Clear chest $C T$ scan and PCR test \\
\hline IRCT20200204046369N1 & Iran & Multi & $\begin{array}{l}\text { Not } \\
\text { available }\end{array}$ & Not available & Not defined & $\mathrm{PAO} 2 / \mathrm{FiO} 2$ through $\mathrm{ABG}$ method \\
\hline IRCT20151227025726N17 & Iran & Single & $\begin{array}{l}\text { Not } \\
\text { available }\end{array}$ & Not available & ARDS & $\begin{array}{l}\text { 1. Daily need for invasive } \\
\text { mechanical ventilation } \\
\text { 2. Death at the end of the study }\end{array}$ \\
\hline IRCT20120225009124N4 & Iran & Single & $\begin{array}{l}\text { Not } \\
\text { available }\end{array}$ & Not available & Not defined & $\begin{array}{l}\text { Improvement in } \mathrm{SpO} 2 \text { measured } \\
\text { by pulse oximeter }\end{array}$ \\
\hline IRCT20200406046963N1 & Iran & Single & $\begin{array}{l}\text { Not } \\
\text { available }\end{array}$ & Not available & $\begin{array}{l}\text { ARDS( acute } \\
\text { respiratory } \\
\text { distress } \\
\text { syndrome) }\end{array}$ & $\begin{array}{l}\text { 1. Mortality rate after } 60 \text { days } \\
\text { 2. Blood } \mathrm{O} 2 \text { saturation } \\
\text { measurement } \\
\text { 3. Need for oxygen therapy }\end{array}$ \\
\hline IRCT20200404046947N1 & Iran & Multi & $\begin{array}{l}\text { Not } \\
\text { available }\end{array}$ & Not available & Not defined & $\begin{array}{l}\text { 1. Findings on the } C T \text { scan } \\
\text { 2. Mortality rate } \\
\text { 3. O2 saturation levels } \\
\text { 4. Need an oxygen therapy at day } \\
3 \text { and discharge time }\end{array}$ \\
\hline IRCT20081027001411N3 & Iran & Multi & $\begin{array}{l}\text { Not } \\
\text { available }\end{array}$ & Not available & ARDS & $\begin{array}{l}\text { 1. Findings on the CT scan } \\
\text { 2. Mortality rate } \\
\text { 3. O2 saturation levels } \\
\text { 4. Need an oxygen therapy at day } \\
3 \text { and discharge time }\end{array}$ \\
\hline IRCT20120215009014N354 & Iran & Single & $\begin{array}{l}\text { Not } \\
\text { available }\end{array}$ & Not available & $\begin{array}{l}\text { Mild-to- } \\
\text { moderate } \\
\text { acute } \\
\text { respiratory } \\
\text { distress } \\
\text { syndrome }\end{array}$ & $\begin{array}{l}\text { 1. Need to mechanical ventilation } \\
\text { 2. The patient's clinical status } \\
\text { 3. Mortality rate }\end{array}$ \\
\hline IRCT20080901001165N52 & Iran & Single & $\begin{array}{l}\text { Not } \\
\text { available }\end{array}$ & Not available & $\begin{array}{l}\text { Moderate to } \\
\text { severe } \\
\text { pneumonia }\end{array}$ & Need to receive ICU service \\
\hline NCT04323592 & Italy & Single & $\begin{array}{l}\text { March } \\
2020\end{array}$ & May 2020 & $\begin{array}{l}\text { Acute } \\
\text { respiratory } \\
\text { distress }\end{array}$ & $\begin{array}{l}\text { 1. Admission to ICU and need for } \\
\text { Invasive mechanical ventilation } \\
\text { 2. In-hospital death within } 28 \text { days }\end{array}$ \\
\hline
\end{tabular}


Table 1 General characteristics of the included trials (Continued)

\begin{tabular}{|c|c|c|c|c|c|c|}
\hline Trial identifier & Country & $\begin{array}{l}\text { Number } \\
\text { of sites }\end{array}$ & Start date & $\begin{array}{l}\text { Expected } \\
\text { completion } \\
\text { date }\end{array}$ & $\begin{array}{l}\text { Stage of } \\
\text { COVID-19 }\end{array}$ & Outcome measures \\
\hline & & & & & syndrome & 3. Endotracheal intubation \\
\hline КСТ0005105 & Korea & Multi & April 2020 & $\begin{array}{l}\text { September } \\
2020\end{array}$ & Mild & $\begin{array}{l}\text { Rate of SARS-CoV-2 eradication at } \\
\text { day } 14 \text { from study enrollment }\end{array}$ \\
\hline IRCT20200318046812N2 & Iran & Multi & $\begin{array}{l}\text { Not } \\
\text { available }\end{array}$ & Not available & Not defined & Admission to intensive care unit \\
\hline NCT04345445 & Malaysia & Single & April 2020 & $\begin{array}{l}\text { October } \\
2020\end{array}$ & Pneumonia & $\begin{array}{l}\text { 1. The proportion of patients } \\
\text { requiring mechanical ventilation } \\
\text { 2. Mean days in ventilation }\end{array}$ \\
\hline NCT04360876 & Not available & Single & May 2020 & $\begin{array}{l}\text { December } \\
2020\end{array}$ & ARDS & Ventilator-free days (VFD) at day 28 \\
\hline NCT04366115 & Not available & $\begin{array}{l}\text { Not } \\
\text { available }\end{array}$ & June 2020 & June 2023 & Not defined & $\begin{array}{l}\text { 1. Dose-limiting toxicities } \\
\text { 2. } 28 \text {-day all-cause mortality for } \\
\text { phases } 1 \text { and } 2\end{array}$ \\
\hline NCT04435795 & Not available & $\begin{array}{l}\text { Not } \\
\text { available }\end{array}$ & June 2020 & March 2021 & Not defined & Improvement in dyspnea at day 7 \\
\hline NCT04355247 & Puerto Rico & Multi & April 2020 & April 2021 & $\begin{array}{l}\text { High-risk } \\
\text { COVID-19 }\end{array}$ & $\begin{array}{l}\text { 1. Clinical complete response } \\
\text { criteria } \\
\text { 2. Need for ventilatory support } \\
\text { 3. O2 Saturation of }>/=93 \% \text { by } \\
\text { day } 14 \text { of therapy } \\
\text { 4. Mortality at day } 28 \\
\text { 5. Findings on CT chest on day } 28\end{array}$ \\
\hline NCT04438980 & Spain & Multi & May 2020 & $\begin{array}{l}\text { February } \\
2021\end{array}$ & Pneumonia & $\begin{array}{l}\text { 1. Proportion of patients } \\
\text { developing treatment failure } \\
\text { 2. Need for mechanical ventilation } \\
\text { 3. Decrease in } \mathrm{SpO} 2<90 \% \text { (in } \\
\text { ambient air) or } \mathrm{PaO} 2<60 \mathrm{mmHg} \\
\text { (in ambient air) or } \mathrm{PaO} 2 \mathrm{FiO} 2<300 \\
\mathrm{mmHg}\end{array}$ \\
\hline NCT04394182 & Spain & Multi & April 2020 & April 2021 & Pneumonia & Oxygen saturation at day 2 \\
\hline NCT04380818 & Spain & Multi & June 2020 & July 2021 & Pneumonia & $\begin{array}{l}\text { Efficacy of low-dose pulmonary ir- } \\
\text { radiation assessed by change in } \\
\text { PAFiO2 by } 20 \%\end{array}$ \\
\hline NCT04355637 & Spain & Multi & April 2020 & $\begin{array}{l}\text { October } \\
2020\end{array}$ & Pneumonia & $\begin{array}{l}\text { Proportion of patients developing } \\
\text { treatment failure }\end{array}$ \\
\hline NCT04341038 & Spain & Single & April 2020 & June 2020 & $\begin{array}{l}\text { Severe lung } \\
\text { injury } \\
\text { secondary to } \\
\text { COVID-19 }\end{array}$ & Time to reach clinical stability \\
\hline NCT04329650 & Spain & Multi & April 2020 & May 2020 & Pneumonia & $\begin{array}{l}\text { Proportion of patients requiring } \\
\text { ICU admission at any time within } \\
\text { the study period }\end{array}$ \\
\hline NCT04325061 & Spain & Multi & April 2020 & $\begin{array}{l}\text { October } \\
2020\end{array}$ & ARDS & 60-day mortality \\
\hline 2020-001827-15 & Spain & Single & $\begin{array}{l}\text { Not } \\
\text { available }\end{array}$ & Not available & Pneumonia & $\begin{array}{l}\text { 1. Proportion of patients with } \\
\text { treatment failure up to } 14 \text { days } \\
\text { after randomization } \\
\text { 2. Mortality rate } \\
\text { 3. ICU admission } \\
\text { 4. Number of patients requiring } \\
\text { mechanical ventilation } \\
\text { 5. Clinical deterioration/worsening, } \\
\text { defined as decrease in } \mathrm{SpO} 2 \\
\text { below } 90 \% \text { or PaO2 below } 60 \\
\mathrm{mmHg} \text { in ambient air }+ \\
\text { radiological progression. }\end{array}$ \\
\hline $2020-001622-64$ & Spain & Single & April 2020 & Not available & Not defined & 1. Measurement of $\mathrm{O} 2$ saturation \\
\hline
\end{tabular}


Table 1 General characteristics of the included trials (Continued)

\begin{tabular}{|c|c|c|c|c|c|c|}
\hline Trial identifier & Country & $\begin{array}{l}\text { Number } \\
\text { of sites }\end{array}$ & Start date & $\begin{array}{l}\text { Expected } \\
\text { completion } \\
\text { date }\end{array}$ & $\begin{array}{l}\text { Stage of } \\
\text { COVID-19 }\end{array}$ & Outcome measures \\
\hline & & & & & & $\begin{array}{l}\text { and/or blood gas, findings on } \\
\text { chest x-ray, CBC, including inflam- } \\
\text { matory markers and blood biomet- } \\
\text { rics, and ECG } \\
\text { 2. 30-day ICU admission and hos- } \\
\text { pital stay } \\
\text { 3. Outbreaks of steroid-related } \\
\text { psychosis }\end{array}$ \\
\hline 2020-001934-37 & Spain & Multi & May 2020 & Not available & Not defined & $\begin{array}{l}\text { 1. Mortality rate } \\
\text { 2. Number of days of ICU stay } \\
\text { 3. Number of patients requiring } \\
\text { non-invasive ventilation (NIV) }\end{array}$ \\
\hline 2020-001413-20 & Spain & Single & April 2020 & Not available & Pneumonia & $\begin{array}{l}\text { Proportion of patients requiring } \\
\text { ICU admission at any time within } \\
\text { the study period }\end{array}$ \\
\hline 2020-001445-39 & Spain & Single & $\begin{array}{l}\text { March } \\
2020\end{array}$ & Not available & Pneumonia & $\begin{array}{l}\text { Time (days) to clinical stability after } \\
\text { initiation of trial treatment for } \\
\text { severe pneumonia secondary to } \\
\text { COVID- } 19 \text { and elevated inflamma- } \\
\text { tory parameters }\end{array}$ \\
\hline 2020-001307-16 & Spain & Single & April 2020 & Not available & ARDS & $\begin{array}{l}\text { Death from any cause in the first } \\
28 \text { days after randomization }\end{array}$ \\
\hline NCT04381364 & Sweden & Multi & May 2020 & $\begin{array}{l}\text { December } \\
2020\end{array}$ & Pneumonia & $\begin{array}{l}\text { Duration of supplemental oxygen } \\
\text { therapy }\end{array}$ \\
\hline NCT04416399 & UK & $\begin{array}{l}\text { Not } \\
\text { available }\end{array}$ & June 2020 & $\begin{array}{l}\text { December } \\
2020\end{array}$ & $\begin{array}{l}\text { Early } \\
\text { infection }\end{array}$ & $\begin{array}{l}\text { Emergency department visit } \\
\text { related to COVID-19 }\end{array}$ \\
\hline NCT04381936 & UK & Multi & $\begin{array}{l}\text { March } \\
2020\end{array}$ & June 2021 & SARS & All-cause mortality \\
\hline NCT04411667 & USA & Multi & April 2020 & $\begin{array}{l}\text { November } \\
2020\end{array}$ & Not defined & $\begin{array}{l}\text { Number of subjects requiring } \\
\text { mechanical ventilation }\end{array}$ \\
\hline NCT04377711 & USA & Multi & June 2020 & $\begin{array}{l}\text { December } \\
2020\end{array}$ & $\begin{array}{l}\text { Symptomatic } \\
\text { COVID-19 } \\
\text { infection }\end{array}$ & $\begin{array}{l}\text { Percentage hospital admission or } \\
\text { death by day } 30\end{array}$ \\
\hline NCT04349410 & USA & $\begin{array}{l}\text { Not } \\
\text { available }\end{array}$ & April 2020 & $\begin{array}{l}\text { November } \\
2020\end{array}$ & Pneumonia & $\begin{array}{l}\text { 1. Improvement in FMTVDM } \\
\text { measurement with nuclear } \\
\text { imaging } \\
\text { 2. Ventilator status } \\
\text { 3. Extubation status } \\
\text { 4. Survival status in } 30 \text { days }\end{array}$ \\
\hline NCT04193878 & USA & Multi & June 2020 & June 2024 & $\begin{array}{l}\text { Pneumonia, } \\
\text { acute } \\
\text { respiratory } \\
\text { failure }\end{array}$ & $\begin{array}{l}\text { Number of patients with acute } \\
\text { respiratory failure (ARF) within } 10 \\
\text { days of randomization }\end{array}$ \\
\hline NCT03852537 & USA & Single & $\begin{array}{l}\text { December } \\
2019\end{array}$ & July 2022 & Pneumonia & $\begin{array}{l}\text { 1. Feasibility of the timely initiation } \\
\text { of corticosteroids and } \\
\text { implementation of biomarker- } \\
\text { titrated corticosteroid dosing } \\
\text { 2. Percentage of eligible patients } \\
\text { adhered to the timely initiation } \\
\text { within } 30 \text { days }\end{array}$ \\
\hline NCT04374071 & USA & Completed & $\begin{array}{l}\text { March } \\
2020\end{array}$ & April 2020 & Pneumonia & $\begin{array}{l}\text { 1. Number of patients transferred } \\
\text { to ICU is each of the group } \\
\text { 2. Number of patients requiring } \\
\text { mechanical ventilation } \\
\text { 3. Mortality rate }\end{array}$ \\
\hline
\end{tabular}


Table 2 Methodological quality of included trials

\begin{tabular}{|c|c|c|c|c|}
\hline Trial identifier & $\begin{array}{l}\text { Estimated sample } \\
\text { size }\end{array}$ & $\begin{array}{l}\text { Allocation (randomized/non- } \\
\text { randomized) }\end{array}$ & $\begin{array}{l}\text { Blinding/ } \\
\text { masking }\end{array}$ & Study design \\
\hline NCT04438980 & 72 & Randomized & Double & Interventional \\
\hline NCT04435795 & 454 & Randomized & Triple & Interventional \\
\hline NCT04425863 & 10 & Not available & Not available & $\begin{array}{l}\text { Non-interventional, prospective } \\
\text { cohort }\end{array}$ \\
\hline NCT04416399 & 478 & Randomized & Open label & Interventional \\
\hline NCT04411667 & 40 & Randomized & Open label & Interventional \\
\hline NCT04395105 & 284 & Randomized & Open label & Interventional \\
\hline NCT04394182 & 15 & Not available & Open label & Interventional \\
\hline NCT04381936 & 12000 & Randomized & Open label & Interventional \\
\hline NCT04348305 & 1000 & Randomized & Quadruple & Interventional \\
\hline NCT04331054 & 436 & Randomized & Open label & Interventional \\
\hline NCT04360876 & 90 & Randomized & Double & Interventional \\
\hline NCT04355247 & 20 & Not available & Open label & Interventional \\
\hline NCT04381364 & 446 & Randomized & Open label & Interventional \\
\hline NCT04380818 & 106 & Non-randomized & Open label & Interventional \\
\hline NCT04377711 & 400 & Randomized & Double & Interventional \\
\hline NCT04377503 & 40 & Randomized & Open label & Interventional \\
\hline NCT04374474 & 75 & Randomized & Open label & Interventional \\
\hline NCT04366115 & 126 & Randomized & Open label & Interventional \\
\hline NCT04361474 & 120 & Randomized & Single & Interventional \\
\hline NCT04359511 & 210 & Randomized & Single & Interventional \\
\hline NCT04355637 & 300 & Randomized & Open label & Interventional \\
\hline NCT04349410 & 500 & Randomized & Single & Interventional \\
\hline NCT04347980 & 122 & Randomized & Single & Interventional \\
\hline NCT04263402 & 100 & Randomized & Single & Interventional \\
\hline NCT04193878 & 600 & Randomized & Triple & Interventional \\
\hline NCT03852537 & 90 & Randomized & Double & Interventional \\
\hline NCT02735707 & 7100 & Randomized & Open label & Randomized, multifactorial trial \\
\hline NCT04345445 & 310 & Randomized & Open label & Interventional \\
\hline NCT04344730 & 550 & Randomized & Quadruple & Interventional \\
\hline NCT04344288 & 304 & Randomized & Open label & Interventional \\
\hline NCT04343729 & 425 & Randomized & Quadruple & Interventional \\
\hline NCT04341038 & 84 & Randomized & Single & Interventional \\
\hline NCT04331470 & 30 & Randomized & Double & Interventional \\
\hline КСТ0005105 & 141 & Randomized & Open label & Interventional \\
\hline NCT04329650 & 200 & Randomized & Open label & Interventional \\
\hline NCT04327401 & 350 & Randomized & Open label & Interventional \\
\hline NCT04325061 & 200 & Randomized & Open label & Interventional \\
\hline NCT04323592 & 173 & Non-randomized & Open label & $\begin{array}{l}\text { Non-interventional, prospective } \\
\text { cohort }\end{array}$ \\
\hline NCT04374071 & 250 & Non-randomized & Not available & $\begin{array}{l}\text { Non-interventional, retrospective } \\
\text { cohort }\end{array}$ \\
\hline NCT04244591 & 80 & Randomized & Open label & Interventional \\
\hline NCT04273321 & 86 & Randomized & Open label & Interventional \\
\hline
\end{tabular}


Table 2 Methodological quality of included trials (Continued)

\begin{tabular}{|c|c|c|c|c|}
\hline Trial identifier & $\begin{array}{l}\text { Estimated sample } \\
\text { size }\end{array}$ & $\begin{array}{l}\text { Allocation (randomized/non- } \\
\text { randomized) }\end{array}$ & $\begin{array}{l}\text { Blinding/ } \\
\text { masking }\end{array}$ & Study design \\
\hline IRCT20080901001165N52 & 50 & Randomized & Open label & Interventional \\
\hline IRCT20200406046963N1 & 40 & Randomized & Open label & Interventional \\
\hline IRCT20200404046947N1 & 68 & Randomized & Single & Interventional \\
\hline IRCT20081027001411N3 & 60 & Randomized & Single & Interventional \\
\hline IRCT20120215009014N354 & 81 & Randomized & Double & Interventional \\
\hline IRCT20200204046369N1 & 48 & Non-randomized & Open label & Interventional \\
\hline IRCT20200318046812N2 & 906 & Randomized & Open label & Interventional \\
\hline IRCT20151227025726N17 & 48 & Randomized & Open label & Interventional \\
\hline IRCT20120225009124N4 & 105 & Randomized & Open label & Interventional \\
\hline ChiCTR2000029386 & 24 & Randomized & Not available & Interventional \\
\hline ChiCTR2000029656 & 50 & Randomized & Open label & Interventional \\
\hline ChiCTR2000030481 & 75 & Randomized (static) & Not available & Interventional \\
\hline 2020-001827-15 & 72 & Randomized & Double & Interventional \\
\hline 2020-001307-16 & 104 & Randomized & Open label & Interventional \\
\hline 2020-001395-15 & 1000 & Randomized & Double & Interventional \\
\hline 2020-001622-64 & 200 & Randomized & Open label & Interventional \\
\hline 2020-001934-37 & 200 & Randomized & Open label & Interventional \\
\hline 2020-001413-20 & 100 & Randomized & Open label & Interventional \\
\hline 2020-001445-39 & 84 & Randomized & Open label & Interventional \\
\hline
\end{tabular}

primary outcomes of the study, blinding, randomization, and study design.

\section{Scrutiny of trials}

Our initial search of the clinical trial registries resulted in 231 trials, of which 167 trials did not satisfy the inclusion criteria and three trials did not have complete data, and after removing the duplicates, 60 trials were included in the final analysis. Thus, 60 trials with 31,732 patients were included in this systematic review. The included trials were classified into trials that included only steroid therapy and those that included steroids in addition to other standard treatment as shown in Table 1.

\section{Type of trials}

Among the included trials, 57 trials were quantitative studies and the remaining three trials were qualitative studies, i.e., non-interventional studies, as shown in the Table 2.

\section{Heterogeneity of trials}

All 60 trials included were heterogenous in that they had various inclusion and exclusion criteria and different treatment protocols for the treatment of various stages of COVID-19. The most common stage of COVID-19 among these trials is pneumonia, which is shown in Table 1.

\section{Methodological quality of the trials}

Among the 60 trials, 54 were randomized. It was unclear how randomization was carried out in three of the trials. Among 54 randomized trials, only 21 trials were blinded, of which 8 were single blinded, 8 were double blinded, 2 were triple blinded, and 3 were quadruple blinded, as shown in Table 2.

\section{Steroid treatment}

Regarding the steroid treatment, the most common steroid used is methylprednisolone (used in 28 trials) at various dosages depending on the age of the patients. Maximum loading dose of methylprednisolone used is $500 \mathrm{mg}$ IV infusion over $1 \mathrm{~h}$ in a trial (IRCT20080901001165N52). Steroids were given from a minimum of 3 days to a maximum of 21 days. Other steroids used are budesonide, ciclesonide, dexamethasone, formoterol, prednisolone, prednisone, and hydrocortisone. In 10 trials, the dose of the steroids used was unclear, and in one trial (ChiCTR2000030481), the treatment regimen was not mentioned. This is shown in Table 3.

Figure 3 depicts the number of trials studying different types of steroids, showing majority of the trials $(N=28)$ have decided to study the effectiveness of methylprednisolone in the treatment of COVID-19. 
Table 3 Steroid treatment in patients with COVID-19

\begin{tabular}{ll}
\hline Trial identifier & Title \\
\hline NCT04425863 & $\begin{array}{l}\text { Evaluation of ivermectin, aspirin, } \\
\text { dexamethasone, and enoxaparin as } \\
\text { treatment of Covid19 } \\
\text { NCT04395105 }\end{array}$ \\
& $\begin{array}{l}\text { Dexamethasone versus usual care } \\
\text { for the treatment of COVID-19 re- } \\
\text { lated ARDS: a multicenter and ran- } \\
\text { domized open-label clinical trial }\end{array}$
\end{tabular}

NCT02735707

NCT04377503

NCT04343729

NCT04327401

NCT04374474

NCT04263402

NCT04244591

NCT04273321
Randomized, embedded, multifactorial adaptive platform trial for community-acquired pneumonia

Comparison of the efficacy and safety of tocilizumab versus methylprednisolone in the cytokine release syndrome of patients with COVID-19. A prospective randomized controlled phase II trial

Methylprednisolone in the treatment of patients with signs of severe acute respiratory syndrome in Covid-19 (MetCOVID)

COVID-19-associated ARDS treated with dexamethasone: Alliance Covid-19 Brasil III (CoDEX)

Olfactory retraining therapy and budesonide nasal rinse for anosmia treatment in patients post-CoVID 19. A randomized controlled trial

An open, prospective/retrospective, randomized controlled cohort study to compare the efficacy of different hormone doses in the treatment of 2019-nCoV severe pneumonia

Glucocorticoid therapy for critically ill patients with severe acute respiratory infections caused by COVID-19: a prospective, randomized controlled trial

Efficacy and safety of corticosteroids Methylprednisolone in COVID-19: a prospective randomized controlled trials

Effectiveness of glucocorticoid therapy in patients with severe

\section{Interventions Dose}

Age (in

years)

Ivermectin; aspirin; dexamethasone; Dexamethasone 4 mg/day IV

$\geq 5$

enoxaparin

Dexamethasone

1. Fixed-duration Hydrocortisone

2. Shock-dependent hydrocortisone

3. Ceftriaxone

4. Moxifloxacin or Levofloxacin

5. Piperacillin-tazobactam

6. Ceftaroline

7. Amoxicillin-clavulanate

8. Macrolide administered for 3-5

days

9. Macrolide administered for up to

14 days

10. Five days of oseltamivir

11. Ten days of oseltamivir

12. Lopinavir/ritonavir

12. Hydroxychloroquine

13. Hydroxychloroquine +

lopinavir/ritonavir

14. Interferon- $\beta 1$ a

15. Anakinra

16. Fixed-duration higher dose

hydrocortisone

17. Tocilizumab

18.Sarilumab

1. Tocilizumab $180 \mathrm{mg} / \mathrm{ml}$

2. Methylprednisolone sodium succinate

Methylprednisolone sodium succinate

Dexamethasone

1. Corticosteroid nasal irrigation 2. Smell household items; olfactory retraining

\section{Methylprednisolone}

Methylprednisolone therapy.

Others: standard care

\section{Methylprednisolone and} intravenous injection
Dexamethasone $16 \mathrm{mg}$ IV OD from $\geq 18$ days 1 to 5 and $8 \mathrm{mg}$ from days 6 to 10

1. Fixed-duration hydrocortisone $50>18$ mg IV q $6 \mathrm{~h} \times 7$ days

2. Shock-dependant hydrocortisone $50 \mathrm{mg}$ IV q $6 \mathrm{~h}$ while in septic shock

3. Fixed-duration higher dose hydrocortisone-100 mg IV every 6 $\mathrm{h} \times 7$ days

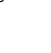


Table 3 Steroid treatment in patients with COVID-19 (Continued)

\begin{tabular}{|c|c|c|c|c|}
\hline Trial identifier & Title & Interventions & Dose & $\begin{array}{l}\text { Age (in } \\
\text { years) }\end{array}$ \\
\hline & $\begin{array}{l}\text { novel coronavirus pneumonia: a } \\
\text { randomized controlled trial }\end{array}$ & & & \\
\hline ChiCTR2000029656 & $\begin{array}{l}\text { A randomized, open-label study to } \\
\text { evaluate the efficacy and safety of } \\
\text { low-dose corticosteroids in hospital- } \\
\text { ized patients with novel coronavirus } \\
\text { pneumonia (COVID-19) }\end{array}$ & Methylprednisolone & Not available & $\geq 18$ \\
\hline ChiCTR2000030481 & $\begin{array}{l}\text { The clinical value of corticosteroid } \\
\text { therapy timing in the treatment of } \\
\text { novel coronavirus pneumonia } \\
\text { (COVID-19): a prospective } \\
\text { randomized controlled trial }\end{array}$ & Not mentioned & Not available & $\geq 18$ \\
\hline NCT04348305 & $\begin{array}{l}\text { Low-dose hydrocortisone in } \\
\text { patients with COVID-19 and severe } \\
\text { hypoxia - the COVID STEROID Trial }\end{array}$ & Hydrocortisone & $\begin{array}{l}\text { Hydrocortisone continuous } \\
\text { infusion: } 200 \mathrm{mg} \text { q } 24 \mathrm{~h} \text { bolus } \\
\text { injections } 50 \mathrm{mg}(10 \mathrm{ml}) \text { every } 6 \mathrm{~h} \\
\times 7 \text { days }\end{array}$ & $\geq 18$ \\
\hline NCT04331054 & $\begin{array}{l}\text { Protective role of inhaled steroids } \\
\text { for Covid-19 infection }\end{array}$ & $\begin{array}{l}\text { 1. Usual practice } \\
\text { 2. Usual practice + Symbicort } \\
\text { Rapihaler }\end{array}$ & $\begin{array}{l}\text { Symbicort (budesonide, formoterol) } \\
200 / 6 \mu \mathrm{g}, 2 \text { puffs bid } \times 30 \text { days }\end{array}$ & $18-75$ \\
\hline NCT04361474 & $\begin{array}{l}\text { A randomized controlled trial } \\
\text { evaluating the efficacy of local } \\
\text { budesonide therapy in the } \\
\text { management of hyposmia in } \\
\text { COVID-19 patients without signs of } \\
\text { severity }\end{array}$ & $\begin{array}{l}\text { 1. Budesonide nasal spray } \\
\text { 2. Physiological serum }\end{array}$ & $\begin{array}{l}\text { Budesonide } 1 \mathrm{mg} / 2 \mathrm{ml} \text { diluted in } \\
250 \mathrm{ml} \text { of physiological saline } 3 \\
\text { syringes of } 20 \mathrm{ml} \text { in each nasal } \\
\text { cavity } \mathrm{BD} \times 30 \text { days }\end{array}$ & $\geq 18$ \\
\hline NCT04359511 & $\begin{array}{l}\text { Efficacy and safety of corticosteroids } \\
\text { in oxygen-dependent patients with } \\
\text { COVID-19 pneumonia in Grand } \\
\text { Ouest Interregion France }\end{array}$ & $\begin{array}{l}\text { 1. Prednisone } \\
\text { 2. Hydrocortisone }\end{array}$ & $\begin{array}{l}\text { Prednisone } 0.7 \mathrm{mg} / \mathrm{kg} / \mathrm{day} \text { PO OD } \\
\times 10 \text { days or hydrocortisone } \\
\text { hemisuccinate } 3.5 \mathrm{mg} / \mathrm{kg} / \text { day } \\
\text { continuous infusion } \times 10 \text { days }\end{array}$ & $\geq 18$ \\
\hline NCT04347980 & $\begin{array}{l}\text { Dexamethasone combined with } \\
\text { hydroxychloroquine compared to } \\
\text { hydroxychloroquine alone for } \\
\text { treatment of severe acute } \\
\text { respiratory distress syndrome } \\
\text { induced by coronavirus disease } 19 \\
\text { (COVID-19): a multicentre, } \\
\text { randomised controlled trial }\end{array}$ & $\begin{array}{l}\text { 1. Dexamethasone and } \\
\text { hydroxychloroquine } \\
\text { 2. Hydroxychloroquine }\end{array}$ & $\begin{array}{l}\text { Dexamethasone } 20 \mathrm{mg} \text { IV OD for } \\
15 \mathrm{~min} \times 5 \text { days followed by } 10 \\
\mathrm{mg} \text { OD } \times 5 \text { days }\end{array}$ & $\geq 18$ \\
\hline NCT04344730 & $\begin{array}{l}\text { Dexamethasone and oxygen } \\
\text { support strategies in ICU patients } \\
\text { with Covid-19 pneumonia } \\
\text { (COVIDICUS) }\end{array}$ & $\begin{array}{l}\text { Dexamethasone injection + } \\
\text { conventional oxygen }\end{array}$ & Dexamethasone $20 \mathrm{mg} / 5 \mathrm{ml} \mathrm{IV}$ & $18-80$ \\
\hline NCT04344288 & $\begin{array}{l}\text { Corticosteroids during Covid-19 viral } \\
\text { pneumonia related to SARS-Cov-2 } \\
\text { infection (CORTI-Covid) }\end{array}$ & Prednisone & $\begin{array}{l}\text { Prednisone } 0.75 \mathrm{mg} / \mathrm{kg} / \text { day } \times 5 \\
\text { days then } 20 \mathrm{mg} / \text { day } \times 5 \text { more } \\
\text { days }\end{array}$ & $\geq 18$ \\
\hline NCT04331054 & $\begin{array}{l}\text { Protective role of inhaled steroids } \\
\text { for Covid-19 infection }\end{array}$ & $\begin{array}{l}\text { 1. Usual practice } \\
\text { 2. Usual practice + Symbicort } \\
\text { Rapihaler }\end{array}$ & $\begin{array}{l}\text { Symbicort (budesonide, formoterol) } \\
200 / 6 \text { mg } 2 \text { puffs bid } \times 30 \text { days }\end{array}$ & $18-75$ \\
\hline NCT04331470 & $\begin{array}{l}\text { Evaluation of efficacy of levamisole } \\
\text { and formoterol + budesonide in } \\
\text { treatment of COVID-19 }\end{array}$ & $\begin{array}{l}\text { 1. Levamisole pill + budesonide + } \\
\text { formoterol inhaler/lopinavir/ } \\
\text { ritonavir + hydroxychloroquine } \\
\text { 2. Lopinavir/ritonavir + } \\
\text { hydoxychloroquine }\end{array}$ & $\begin{array}{l}\text { Budesonide }+ \text { formoterol } \\
\text { inhalation } 1-2 \text { puffs q } 12 \mathrm{~h}\end{array}$ & $15-100$ \\
\hline IRCT20080901001165N52 & $\begin{array}{l}\text { Investigating the efficacy of high } \\
\text { dose of glucocorticoid in patients } \\
\text { with moderate to severe } \\
\text { pneumonia related to COVID-19 }\end{array}$ & $\begin{array}{l}\text { Methylprednisolone and } \\
\text { prednisolone }\end{array}$ & $\begin{array}{l}\text { Day 1: Amp. methylprednisolone } \\
500 \mathrm{mg} \text { IV infusion over } 1 \text { hour. } \\
\text { At days } 2 \text { and 3: Amp. } \\
\text { methylprednisolone } 250 \mathrm{mg} \mathrm{IV} \\
\text { infusion over } 1 \mathrm{~h} . \\
\text { At days } 4 \text { and 5: Amp. } \\
\text { methylprednisolone } 100 \mathrm{mg} \mathrm{IV} \\
\text { infusion over } 1 \mathrm{~h} \text {. } \\
\text { Then, tab. prednisolone } 25 \mathrm{mg} \text { PO }\end{array}$ & $18-85$ \\
\hline
\end{tabular}


Table 3 Steroid treatment in patients with COVID-19 (Continued)

\begin{tabular}{|c|c|}
\hline Trial identifier & Title \\
\hline IRCT20200204046369N1 & $\begin{array}{l}\text { Evaluation of methylprednisolone } \\
\text { administration as a therapeutic } \\
\text { option in the } 2019 \text { novel } \\
\text { coronavirus (COVID-19): a non- } \\
\text { randomized controlled study }\end{array}$ \\
\hline IRCT20200318046812N2 & $\begin{array}{l}\text { Safety and efficacy of } \\
\text { "Hydroxychloroquine + } \\
\text { Azithromycin + naproxen + } \\
\text { Prednisolone" and } \\
\text { "Hydroxychloroquine + } \\
\text { Azithromycin + naproxen" regimens } \\
\text { in comparison with } \\
\text { "Hydroxychloroquine + kaletra" on } \\
\text { the need for intensive care unit } \\
\text { treatment in patients with COVID- } \\
\text { 19; a randomized, multicenter, } \\
\text { parallel }\end{array}$ \\
\hline
\end{tabular}

IRCT20151227025726N17 Evolution of the efficacy and safety of Dexamethasone administration in patients with mild to moderate COVID-19 acute respiratory disease syndrome

IRCT20120225009124N4 Efficacy of different methods of administration of combination regimen including dexamethasone, IV-IG and interferon beta for treatment of patients with severe COVID-19: a randomized controlled trial

IRCT20200406046963N1 Evaluation of the efficacy and safety of methylprednisolone pulse therapy in treatment of COVID-19 patients with ARDS.

IRCT20200404046947N

Study of methylprednisolone effects on treatment and clinical symptoms and laboratory signs of Iranian COVID-19 patients: a clinical trial study

IRCT20081027001411N3 Study of prednisolone effects on treatment and clinical symptoms and laboratory signs of Iranian COVID-19 patients: a clinical trial study

Evaluating the effect of intravenous

IRCT20120215009014N354 hydrocortisone, methylprednisolone, and dexamethasone in treatment of patients with moderate to severe acute respiratory distress syndrome caused by COVID-19: a double blind randomized clinical trial

NCT04323592

Methylprednisolone for patients with COVID-19 severe acute respiratory syndrome (MP-C19)

КСT0005105

A trial of ciclesonide in adults with

\section{Interventions}

Methylprednisolone

Hydroxychloroquine, azithromycin, naproxen, prednisolone

Dexamethas mild COVID-19

\section{Dose}

Age (in

years)

daily until the day of discharge,

then tab. prednisolone will

gradually tapered off over 1 month

Methylprednisolone 20 mg/day

Prednisolone five $5 \mathrm{mg}$ tablets a

$16-100$ day $\times 5$ days
Dexamethasone

Methylprednisolone

Methylprednisolone

Prednisolone

Hydrocortisone,

methylprednisolone, and

dexamethasone

Dexamethasone $20 \mathrm{mg}$ IV days 1- $\quad \geq 18$ 5 , then $10 \mathrm{mg}$ days $6-10$

Not available

Methylprednisolone $1000 \mathrm{mg}$ for 3 18-90 days

Methylprednisolone $250 \mathrm{mg}$ for $3 \geq 18$ days

Prednisolone $0.5 \mathrm{mg} / \mathrm{kg}$ in three divided doses up to $30 \mathrm{mg}$ per day for 5-7 days

Group 1: Hydrocortisone $50 \mathrm{mg}$ IV $18-70$

q. $6 \mathrm{~h} \times 5$ days Group 2:

Methylprednisolone $40 \mathrm{mg} \mathrm{IV} \mathrm{q} 12$ $\mathrm{h} \times 5$ days

Group 3: Dexamethasone IV 20 mg daily $\times 5$ days

Methylprednisolone and other standard care

1. Ciclesonide (Alvesco $\left.{ }^{\circledR}\right) 320 \mu \mathrm{g}$ inhalation twice a day for 14 days 2. Ciclesonide $\left(\right.$ Alvesco $\left.^{\circledR}\right) 320 \mu \mathrm{g}$ inhalation twice a day for 14 days + hydroxychloroquine 400 mg per
Methylprednisolone 80 mg/kg IV bolus

Ciclesonide (Alvesco $\left.{ }^{\circledast}\right) 320 \mu \mathrm{g}$

inhalation $\mathrm{BD} \times 14$ days 
Table 3 Steroid treatment in patients with COVID-19 (Continued)

\begin{tabular}{|c|c|c|c|c|}
\hline Trial identifier & Title & Interventions & Dose & $\begin{array}{l}\text { Age (in } \\
\text { years) }\end{array}$ \\
\hline & & day for 10 days & & \\
\hline NCT04345445 & $\begin{array}{l}\text { Study to evaluate the efficacy and } \\
\text { safety of tocilizumab versus } \\
\text { corticosteroids in hospitalized } \\
\text { COVID-19 patients with high risk of } \\
\text { progression }\end{array}$ & $\begin{array}{l}\text { 1. Tocilizumab IV } \\
\text { 2. Methylprednisolone IV }\end{array}$ & $\begin{array}{l}\text { Methylprednisolone } 120 \text { mg/day } \\
\text { for } 3 \text { days }\end{array}$ & $18-80$ \\
\hline NCT04435795 & $\begin{array}{l}\text { Ciclesonide clinical trial for COVID- } \\
19 \text { treatment }\end{array}$ & Ciclesonide & $\begin{array}{l}\text { Ciclesonide } 600 \mu \mathrm{g} \text { BID inhaled } \\
\text { with aerochamber + Nasal } \\
\text { ciclesonide } 200 \mu \mathrm{g} \text { DIE }\end{array}$ & $\geq 18$ \\
\hline NCT04360876 & $\begin{array}{l}\text { Targeted steroids for ARDS due to } \\
\text { COVID-19 pneumonia: a pilot ran- } \\
\text { domized clinical trial }\end{array}$ & $\begin{array}{l}\text { 1. Dexamethasone injection } \\
\text { 2. Placebo }\end{array}$ & $\begin{array}{l}\text { Dexamethasone } 20 \mathrm{mg} \mathrm{IV} \mathrm{OD} 5 \\
\text { days followed by } 10 \mathrm{mg} \mathrm{OD} \times 5 \\
\text { days }\end{array}$ & $\geq 18$ \\
\hline NCT04366115 & $\begin{array}{l}\text { A randomized, double-blind, } \\
\text { placebo-controlled, phase } 1 / 2 \text { study } \\
\text { evaluating AVM0703 in patients } \\
\text { with COVID-19 }\end{array}$ & $\begin{array}{l}\text { 1. AVM0703 } \\
\text { 2. Placebo } \\
\text { 3. Hydrocortisone }\end{array}$ & $\begin{array}{l}\text { 1. AVM0703 (dexamethasone } \\
\text { sodium phosphate) } 10 \mathrm{mg} / \mathrm{ml} \\
\text { single IV infusion in NS over } 1 \text { hour } \\
\text { 2. Hydrocortisone dose not } \\
\text { available }\end{array}$ & $\geq 18$ \\
\hline NCT04355247 & $\begin{array}{l}\text { Prophylactic corticosteroid to } \\
\text { prevent COVID-19 cytokine storm }\end{array}$ & $\begin{array}{l}\text { Methylprednisolone } 80 \mathrm{mg} / \mathrm{ml} \\
\text { injectable suspension }\end{array}$ & $\begin{array}{l}\text { Methylprednisolone } 80 \mathrm{mg} \text { IV bolus } \\
\text { injection OD } \times 5 \text { days }\end{array}$ & $\geq 18$ \\
\hline NCT04438980 & $\begin{array}{l}\text { Treatment of COVID-19 pneumonia } \\
\text { with glucocorticoids. A randomized } \\
\text { controlled trial }\end{array}$ & $\begin{array}{l}\text { 1. Methylprednisolone } \\
\text { 2. Placebo }\end{array}$ & $\begin{array}{l}\text { Methylprednisolone } 120 \mathrm{mg} / \text { day IV } \\
\text { infusion } \times 3 \text { days }\end{array}$ & $18-80$ \\
\hline NCT04394182 & $\begin{array}{l}\text { Low doses of lung radiation therapy } \\
\text { in cases of COVID- } 19 \text { pneumonia: } \\
\text { prospective multicentric study in ra- } \\
\text { diation oncology centers }\end{array}$ & $\begin{array}{l}\text { 1. Ultra-low-dose radiotherapy } \\
\text { 2. Ventilatory support with oxygen } \\
\text { therapy } \\
\text { 3. Lopinavir/ritonavir, } \\
\text { hydroxychloroquine, azithromycin, } \\
\text { piperacillin/tazobactam, Low } \\
\text { molecular weight heparin, } \\
\text { corticosteroid injection, tocilizumab }\end{array}$ & $\begin{array}{l}\text { Methylprednisolone } 250 \mathrm{mg} \times 3 \\
\text { boluses }\end{array}$ & $18-120$ \\
\hline NCT04380818 & $\begin{array}{l}\text { Low dose anti-inflammatory radio- } \\
\text { therapy for the treatment of pneu- } \\
\text { monia by COVID-19: multi-central } \\
\text { prospective study }\end{array}$ & $\begin{array}{l}\text { 1. Low-dose radiotherapy; hydroxy- } \\
\text { chloroquine Sulfate } \\
\text { 2. Ritonavir/lopinavir } \\
\text { 3. Tocilizumab Injection (Actemra) } \\
\text { 4. Azithromycin } \\
\text { 5. Corticosteroid } \\
\text { 6. Low molecular weight heparin; } \\
\text { oxygen supply }\end{array}$ & Not available & 18-99 \\
\hline NCT04355637 & $\begin{array}{l}\text { Treatment with inhaled } \\
\text { corticosteroids in patients } \\
\text { hospitalized because of COVID19 } \\
\text { pneumonia }\end{array}$ & Inhaled budesonide & Not available & $18-79$ \\
\hline NCT04341038 & $\begin{array}{l}\text { Clinical trial to evaluate } \\
\text { methylprednisolone pulses and } \\
\text { tacrolimus in patients with COVID- } \\
19 \text { lung injury (TACROVID) }\end{array}$ & $\begin{array}{l}\text { 1. Tacrolimus } \\
\text { 2. Methylprednisolone }\end{array}$ & $\begin{array}{l}\text { Methylprednisolone } 120 \mathrm{mg} \text { daily } \\
\times 3 \text { days }\end{array}$ & $\begin{array}{l}\text { Not } \\
\text { available }\end{array}$ \\
\hline NCT04329650 & $\begin{array}{l}\text { Efficacy and safety of siltuximab vs. } \\
\text { corticosteroids in hospitalized } \\
\text { patients with COVID-19 pneumonia }\end{array}$ & $\begin{array}{l}\text { 1. Siltuximab } \\
\text { 2. Methylprednisolone }\end{array}$ & Methylprednisolone 250 mg/24 h & $\geq 18$ \\
\hline NCT04325061 & $\begin{array}{l}\text { Efficacy of dexamethasone } \\
\text { treatment for patients with ARDS } \\
\text { caused by COVID-19 (DEXA- } \\
\text { coVID19) }\end{array}$ & Dexamethasone & $\begin{array}{l}\text { Dexamethasone } 20 \mathrm{mg} / \mathrm{IV} / \text { daily } \times 5 \\
\text { days }\end{array}$ & $>18$ \\
\hline 2020-001827-15 & $\begin{array}{l}\text { Early treatment of pneumonia } \\
\text { Covid- } 19 \text { with glucocorticoids. ran- } \\
\text { domized controlled clinical trial }\end{array}$ & $\begin{array}{l}\text { Methylprednisolone and } \\
\text { hydroxychloroquine }\end{array}$ & Not available & $\geq 18$ \\
\hline $2020-001622-64$ & $\begin{array}{l}\text { Outpatient treatment of Covid-19 } \\
\text { with early pulmonary corticosteroids } \\
\text { as an opportunity to modify the }\end{array}$ & Prednisone & Not available & $18-74$ \\
\hline
\end{tabular}


Table 3 Steroid treatment in patients with COVID-19 (Continued)

\begin{tabular}{|c|c|c|c|c|}
\hline Trial identifier & Title & Interventions & Dose & $\begin{array}{l}\text { Age (in } \\
\text { years) }\end{array}$ \\
\hline & course of the disease & & & \\
\hline 2020-001934-37 & $\begin{array}{l}\text { Use of corticosteroids in patients } \\
\text { with SARS-CoV2 coronavirus infec- } \\
\text { tion (glucocovid) pragmatic trial } \\
\text { inserted in real practice during a } \\
\text { pandemic covid-19 }\end{array}$ & Methylprednisolone & Not available & $18-85$ \\
\hline 2020-001413-20 & $\begin{array}{l}\text { Phase 2, randomized, open-label } \\
\text { study to compare the efficacy and } \\
\text { safety of siltuximab vs. corticoste- } \\
\text { roids in hospitalized patients with } \\
\text { COVID-19 pneumonia }\end{array}$ & Methylprednisolone and siltuximab & Not available & $\geq 18$ \\
\hline 2020-001445-39 & $\begin{array}{l}\text { Pragmatic, controlled, open, single } \\
\text { center, randomized, phase li clinical } \\
\text { trial to evaluate methylprednisolone } \\
\text { pulses and tacrolimus in } \\
\text { hospitalized patients with severe } \\
\text { pneumonia secondary to COVID-19. }\end{array}$ & $\begin{array}{l}\text { 1. Methylprednisolone } \\
\text { 2. Tacrolimus }\end{array}$ & Not available & $\geq 18$ \\
\hline 2020-001307-16 & $\begin{array}{l}\text { Efficacy and safety of corticoids in } \\
\text { patients with adult respiratory } \\
\text { distress syndrome (ARDS) secondary } \\
\text { to COVID-19. }\end{array}$ & Methylprednisolone hemisuccinate & Not available & $\geq 18$ \\
\hline NCT04381364 & $\begin{array}{l}\text { Inhalation of ciclesonide for patients } \\
\text { with COVID-19: a randomised open } \\
\text { treatment study (HALT COVID-19) }\end{array}$ & Ciclesonide inhalation & $\begin{array}{l}\text { Ciclesonide inhalation } 320 \mu \mathrm{g} \mathrm{BD} \times \\
14 \text { days }\end{array}$ & $18-84$ \\
\hline NCT04416399 & $\begin{array}{l}\text { Use of high dose inhaled } \\
\text { corticosteroids as treatment of early } \\
\text { COVID-19 infection to prevent clin- } \\
\text { ical deterioration and hospitalization }\end{array}$ & Budesonide dry powder inhaler & $\begin{array}{l}\text { Budesonide } 400 \mu \mathrm{g} \text { per inhalation, } \\
2 \text { inhalations twice a day } \times 28 \text { days }\end{array}$ & $>18$ \\
\hline NCT04381936 & $\begin{array}{l}\text { Randomized evaluation of COVID-19 } \\
\text { therapy }\end{array}$ & $\begin{array}{l}\text { 1. Lopinavir-ritonavir } \\
\text { 2. Dexamethasone/prednisolone } \\
\text { 3. Hydroxychloroquine } \\
\text { 4. Azithromycin } \\
\text { 5. Biological: convalescent plasma } \\
\text { 6. Tocilizumab }\end{array}$ & $\begin{array}{l}\text { Dexamethasone } 6 \mathrm{mg} \text { PO OD } \times 10 \\
\text { days }\end{array}$ & $\begin{array}{l}\text { Child, } \\
\text { adult, } \\
\text { older } \\
\text { adult }\end{array}$ \\
\hline NCT04411667 & $\begin{array}{l}\text { Randomized open label study of } \\
\text { standard of care plus intravenous } \\
\text { immunoglobulin (IVIG) compared to } \\
\text { standard of care alone in the } \\
\text { treatment of COVID-19 infection }\end{array}$ & $\begin{array}{l}\text { IVIG (Octagam) premedication and } \\
\text { methylprednisolone }\end{array}$ & $\begin{array}{l}\text { Methylprednisolone } 40 \mathrm{mg} \text { IV push } \\
\times 130-50 \text { min before each IVIG } \\
\text { infusion }\end{array}$ & $\geq 18$ \\
\hline NCT04377711 & $\begin{array}{l}\text { A phase 3, multicenter, randomized, } \\
\text { double-blind, placebo-controlled } \\
\text { study to assess the safety and effi- } \\
\text { cacy of ciclesonide metered-dose } \\
\text { inhaler in non-hospitalized patients } \\
12 \text { years of age and older with } \\
\text { symptomatic COVID-19 infection }\end{array}$ & $\begin{array}{l}\text { 1. Ciclesonide } \\
\text { 2. Placebo }\end{array}$ & $\begin{array}{l}\text { Alvesco (ciclesonide) } 320 \mu \mathrm{g} \text { b.i.d. } \\
\times 30 \text { days via pMDI }\end{array}$ & $12-100$ \\
\hline NCT04349410 & $\begin{array}{l}\text { The fleming [FMTVDM] directed } \\
\text { CoVid-19 treatment protocol }\end{array}$ & $\begin{array}{l}\text { 1. Hydroxychloroquine, } \\
\text { azithromycin } \\
\text { 2. Hydroxychloroquine, doxycycline } \\
\text { 3. Hydroxychloroquine, clindamycin } \\
\text { 4. Hydroxychloroquine, } \\
\text { clindamycin, primaquine_low } \\
\text { dose } \\
\text { 5. Hydroxychloroquine, } \\
\text { clindamycin, primaquine_-high } \\
\text { dose } \\
\text { 6. Remdesivir } \\
\text { 7. Tocilizumab } \\
\text { 8. Methylprednisolone } \\
\text { 9. Interferon-Alpha2B } \\
\text { 10: Losartan plus convalescent } \\
\text { serum }\end{array}$ & $\begin{array}{l}\text { Methylprednisolone } 80 \mathrm{mg} \text { IV over } \\
30 \text { min b.i.d. } \times 7 \text { days, then taper } \\
\text { off }\end{array}$ & $\begin{array}{l}\text { Child, } \\
\text { adult, } \\
\text { older } \\
\text { adult }\end{array}$ \\
\hline
\end{tabular}


Table 3 Steroid treatment in patients with COVID-19 (Continued)

\begin{tabular}{|c|c|c|c|c|}
\hline Trial identifier & Title & Interventions & Dose & $\begin{array}{l}\text { Age (in } \\
\text { years) }\end{array}$ \\
\hline NCT04193878 & $\begin{array}{l}\text { Arrest respiratory failure from } \\
\text { pneumonia (Arrest pneumonia) }\end{array}$ & $\begin{array}{l}\text { 1. Inhaled budesonide and } \\
\text { formoterol } \\
\text { 2. Inhaled placebo }\end{array}$ & $\begin{array}{l}\text { Formoterol aerosolized }-20 \mu \mathrm{g} / 2 \\
\mathrm{ml} \text { ) and budesonide }-1.0 \mathrm{mg} / 2 \mathrm{ml} \\
\mathrm{q} 12 \mathrm{~h} \times 14 \text { doses }\end{array}$ & $\geq 18$ \\
\hline NCT03852537 & $\begin{array}{l}\text { SMART Trial: steroid dosing by } \\
\text { biomarker guided titration in } \\
\text { critically ill patients with pneumonia }\end{array}$ & $\begin{array}{l}\text { Methylprednisolone. Other: usual } \\
\text { care }\end{array}$ & $\begin{array}{l}\text { Methylprednisolone- } \\
\text { predetermined dosing table-- } \\
\text { discontinue if CR }<0.5 \mathrm{mg} ; 0.5 \mathrm{mg} \\
\text { if CRP is } 51-100 \mathrm{mmol} / \mathrm{L} \text { or } 0.75 \\
\mathrm{mg} / \mathrm{kg} \text { if CRP level is } 101-150 \\
\mathrm{mmol} / \mathrm{L} ; 1 \mathrm{mg} / \mathrm{kg} \text { if CRP } 151-200 \\
\mathrm{mmol} / \mathrm{L} \text { or } 1.5 \mathrm{mg} / \mathrm{kg} \text { if CRP level > } \\
200 \mathrm{mmol} / \mathrm{L} \text { or dose equivalent of } \\
\text { oral prednisone for the above }\end{array}$ & $\geq 18$ \\
\hline NCT04374071 & $\begin{array}{l}\text { Early short course corticosteroids in } \\
\text { hospitalized patients with COVID-19 }\end{array}$ & Methylprednisolone & $\begin{array}{l}\text { 1. Methylprednisolone } 0.5 \text { to } 1 \mathrm{mg} / \\
\mathrm{kg} / \text { day IV in two divided doses } \times 3 \\
\text { days } \\
\text { 2. Hydroxychloroquine and IV } \\
\text { methylprednisolone } 0.5 \text { to } 1 \mathrm{mg} / \\
\mathrm{kg} / \text { day in } 2 \text { divided doses } \times 3-7 \\
\text { days }\end{array}$ & $\geq 18$ \\
\hline
\end{tabular}

\section{Primary and secondary outcomes}

Table 1 summarizes results from all 60 studies. All the trials had clearly defined primary and secondary outcomes of interest, in which only 11 trials had evaluation of respiratory rate as one of their outcomes. Common outcomes measured are respiratory rate, mortality rate, ventilation free days, days in ICU, patient Sequential Organ Failure Assessment (SOFA) score, Murray lung injury score, National Early Warning Score 2 (NEWS2) score, number of patients with treatment failure, rate of remission and progression, blood oxygen saturation, chest $\mathrm{x}$-ray, steroidrelated adverse effect, and toxicity monitoring. Table 4 summarizes the consolidation of completed trials with results. All the completed trials have used methylprednisone, dexamethasone, and hydrocortisone as drug of choices.

The data obtained from this review shows that steroids of different doses and types were included in numerous ongoing clinical trials. Their safety and efficacy in

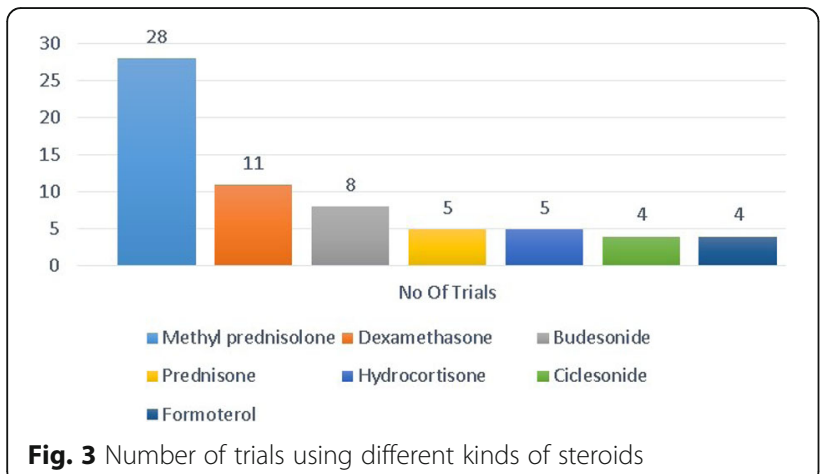

Fig. 3 Number of trials using different kinds of steroids managing symptoms of COVID-19, especially in the pneumonia stage, were tested. The trials also included patients of different age groups at different stages of COVID-19. The COVID-19 infection goes through three stages from asymptomatic phase to ARDS (acute respiratory distress syndrome) phase. The 2019-nCoV, after entering the nasal cavity, adheres to the epithelial cells and binds to ACE2 receptor [24]. Owing to this reason, it may be evident that different corticosteroids act through different mechanisms to minimize the symptoms of COVID-19 infection. Table 3 represents the total number of population recruited in each trial, from which we estimate the total ARDS population recruited to be 3880 patients with disease stages ranging from moderate to severe respiratory distress of which methylprednisolone was the most commonly used corticosteroids. A study by H.P. Wiedemann et al. showed that methylprednisolone increased mortality rates by at least 14 days after the onset of ARDS, which gives an impression that the routine use of methylprednisolone is not effective in ARDS [25]. Another study by Nelson Lee et al. shows that SARS-CoV RNA concentrations in the second and third week of illness were significantly higher in patients who received early hydrocortisone treatment compared to placebo; thus, it is recommended to be avoided, but can be cautiously used in SARS [26]. The potential risks associated with high-dose corticosteroids in treating 2019-nCoV pneumonia include secondary infections, long-term complications, and prolonged virus shedding and escalating towards advanced stages [27]. Another study conducted by G.C. Khilnani and H. Vijay registered increased mortality rate $(35.7 \%)$ with the use of corticosteroids [28-33]. Positively, the RECOVERY trial (Randomised Evaluation of COVID-19 therapy) 
Table 4 Characteristics of published completed trials

\begin{tabular}{|c|c|c|c|c|c|}
\hline Ref & Country & $\begin{array}{l}\text { Year of } \\
\text { publication }\end{array}$ & Steroid used & Primary outcomes & $P$ value \\
\hline$[24]$ & China & 2020 & $\begin{array}{l}\text { Methylprednisolone } 396 \text { of } 409 \\
\text { [96.8\%], dexamethasone } \\
32 \text { of } 409 \text { [7.8\%] patients- } \\
\text { hydrocortisone equivalent }\end{array}$ & $\begin{array}{l}\text { Corticosteroid therapy had higher 28-day } \\
\text { mortality rate. Delay in SARS-CoV RNA } \\
\text { clearance }(P=0.00017)\end{array}$ & $<0.05$ \\
\hline$[25]$ & USA & 2020 & $\begin{array}{l}\text { Hydrocortisone } 200 \text { mg/day } \\
\text { and tapered } \\
\text { till } 50 \mathrm{mg} / \text { day }\end{array}$ & $\begin{array}{l}\text { Treatment failure occurred in hydrocortisone } \\
\text { patient is } 42.1 \% \text { compared to placebo } 50.7 \%\end{array}$ & $<0.045$ \\
\hline$[26]$ & Netherlands & 2020 & $\begin{array}{l}\text { Methylprednisolone } 80 \text { mg, } \\
250 \mathrm{mg}\end{array}$ & $\begin{array}{l}\text { There was a } 79 \% \text { higher likelihood of two stage } \\
\text { improvement in respiratory status }\end{array}$ & $<0.025$ \\
\hline$[27]$ & USA & 2020 & Hydrocortisone 50 mg, 100 mg & $\begin{array}{l}\text { The in-hospital death in treatment group is } 30 \% \\
\text { and } 26 \% \text { compared to no hydrocortisone, i.e. } 33 \%\end{array}$ & $<0.05$ \\
\hline
\end{tabular}

concluded that in hospitalized patients with COVID-19, corticosteroid reduced 28-day mortality among those receiving invasive mechanical ventilation or oxygen at randomization, but not among patients not receiving respiratory support [34]. Moreover, excessive levels of glucocorticoids have shown to precipitate heart failure by aggravating fluid retention, triggering risk factors like glucose intolerance and dyslipidemia, and by worsening atheromatous vascular disease. Additionally, increased risk of mortality with high serum levels of cortisol have been reported, further establishing a link between use of corticosteroids and increased heart failure risk [35]. Thus, the usage of corticosteroids at various stages of COVID-19 is still questionable with higher mortality rates than the comparator. More information can be gained from results from the completed trials. Though four trials have completed its recruitment, results were not available in the registry. The completed four trials were registered in the Iranian clinical trial registry. The outcomes measured in these trials were mortality rate, need for ICU services, duration of stay in the hospital, assessment of side effects, readmission rate, need for oxygen therapy, blood $\mathrm{O}_{2}$, levels, chest $\mathrm{x}$-ray, PAO2/fio2, and need for invasive mechanical ventilation and intubation.

\section{Conclusion}

Numerous interventional and non-interventional studies are being conducted to study the efficacy of corticosteroids in COVID-19. Corticosteroids can regulate immune-mediated lung injury and decrease the development to respiratory failure and death. Dexamethasone has been reported to reduce the duration of mechanical ventilation. Long-term glucocorticoid therapy has displayed significant improvement in indices of alveolarcapillary membrane permeability and mediators of inflammation and tissue repair. Few preliminary trial findings show promising results and recommend the use of methylprednisolone and dexamethasone in the severe form of the COVID-19. Few studies have reported that early administration of dexamethasone could reduce duration of mechanical ventilation and overall mortality in patients with established moderate to severe ARDS; however, there is insufficient data to prove its benefits over its risk. Routine use of corticosteroids should be favored only after a better insight is obtained, with the completion of these trials.

\section{Abbreviations \\ ACE: Angiotensin-converting enzyme; ARDS: Acute respiratory distress syndrome; ChiCTR: Chinese Clinical Trial Registry; COVID-19: Coronavirus disease 2019; CRiS: Clinical Research Information Service-Republic of Korea; EU: European Union; ICU: Intensive care unit; IRCT: Iranian Registry of Clinical Trials; MERS-CoV: Middle East respiratory syndrome coronavirus; NEWS2: National Early Warning Score 2; RNA: Ribonucleic acid; SARS- CoV: Severe acute respiratory syndrome coronavirus; SCAP: Severe community-acquired pneumonia; SOFA: Sequential Organ Failure Assessment; WHO: World Health Organization}

\section{Acknowledgements \\ Not applicable}

Authors' contributions

RR and PSB contributed in the literature search, data collection, data analysis, and writing. PV did the data analysis, data interpretation, figures, and writing. SJUCJ is responsible for the concept, design, methods, data interpretation, writing, and proof reading. The authors read and approved the final manuscript.

Funding

Not applicable.

Availability of data and materials

The datasets analyzed during the current study will be available from the corresponding author on reasonable request.

\section{Declarations}

Ethics approval and consent to participate Not applicable.

Consent for publication

Not applicable.

Competing interests

The authors declare that they have no competing interests. 
Received: 31 July 2020 Accepted: 25 February 2021

Published online: 17 March 2021

\section{References}

1. Zhu N, Zhang D, Wang W, Li X, Yang B, Song J, Zhao X, Huang B, Shi W, Lu R, Niu P, Zhan F, Ma X, Wang D, Xu W, Wu G, Gao GF, Tan W, China Novel Coronavirus Investigating and Research Team (2020) A novel coronavirus from patients with pneumonia in China, 2019. N Engl J Med 382(8):727-733. https://doi.org/10.1056/NEJMoa2001017

2. Gorbalenya AE, Baker SC, Baric RS, de Groot RJ, Drosten C, Gulyaeva AA, Haagmans BL, Lauber C, Leontovich AM, Neuman BW, Penzar D, Perlman S, Poon LLM, Samborskiy D, Sidorov IA, Sola I, Ziebuhr J (2020) Severe acute respiratory syndrome-related coronavirus: The species and its viruses - a statement of the Coronavirus Study Group. Nat Microbiol 5:536-544. https:// doi.org/10.1038/s41564-020-0695-z

3. WHO. WHO Director-General's remarks at the media briefing on 2019-nCoV on 11 February 2020. Available from: https://www.who.int/zh/dg/speeches/ detail/who-director-general-s-remarks-at-the-media-briefing-on-2019-ncovon-11-february-2020. Accessed 25 June 2020.

4. He F, Deng Y, Li W (2020) Coronavirus disease 2019 (COVID-19): what we know? J Med Virol 92(7):719-725. https://doi.org/10.1002/jmv.25766

5. Huang C, Wang Y, Li X, Ren L, Zhao J, Hu Y, Zhang L, Fan G, Xu J, Gu X, Cheng Z, Yu T, Xia J, Wei Y, Wu W, Xie X, Yin W, Li H, Liu M, Xiao Y, Gao H, Guo L, Xie J, Wang G, Jiang R, Gao Z, Jin Q, Wang J, Cao B (2020) Clinical features of patients infected with 2019 novel coronavirus in Wuhan, China. Lancet 395(10223):497-506. https://doi.org/10.1016/S0140-6736(20)30183-5

6. Chan JF, Yuan S, Kok KH, To KK, Chu H, Yang J, Xing F, Liu J, Yip CC, Poon RW, Tsoi HW, Lo SK, Chan KH, Poon VK, Chan WM, Ip JD, Cai JP, Cheng VC, Chen H, Hui CK, Yuen KY (2020) A familial cluster of pneumonia associated with the 2019 novel coronavirus indicating person-to-person transmission: a study of a family cluster. Lancet 395(10223):514-523. https://doi.org/10.101 6/S0140-6736(20)30154-9

7. https://www.who.int/emergencies/diseases/novel-coronavirus-2019/events-a s-they-happen (Accessed 29 June 2020)

8. Ahn DG, Shin HJ, Kim MH, Lee S, Kim HS, Myoung J, Kim BT, Kim SJ (2020) Current status of epidemiology, diagnosis, therapeutics, and vaccines for novel coronavirus disease 2019 (COVID-19). J Microbiol Biotechnol 30(3): 313-324. https://doi.org/10.4014/jmb.2003.03011

9. Lu H (2020) Drug treatment options for the 2019-new coronavirus (2019nCoV). Biosci Trends 14(1):69-71. https://doi.org/10.5582/bst.2020.01020

10. Cava C, Bertoli G, Castiglioni I (2020) In silico discovery of candidate drugs against Covid-19. Viruses 12(4):404. https://doi.org/10.3390/v12040404

11. Kleandrova W, Speck-Planche A (2020) The QSAR paradigm in fragmentbased drug discovery: from the virtual generation of target inhibitors to multi-scale modeling. Mini reviews in medicinal chemistry 20(14):1357-1374. https://doi.org/10.2174/1389557520666200204123156

12. Redkar S, Mondal S, Joseph A, Hareesha KS (2020) A machine learning approach for drug-target interaction prediction using wrapper feature selection and class balancing. Molecular informatics 39(5):e1900062. https:// doi.org/10.1002/minf.201900062

13. Singh R, Bhardwaj V, Das P, Purohit R (2019) Natural analogues inhibiting selective cyclin-dependent kinase protein isoforms: a computational perspective. J Biomol Struct Dyn 4:1-10. https://doi.org/10.1080/07391102.2 019.1696709

14. Liu B, He H, Luo H, Zhang T, Jiang J (2019) Artificial intelligence and big data facilitated targeted drug discovery. Stroke Vasc Neurol 4(4):206-213. https://doi.org/10.1136/svn-2019-000290

15. Martinez-Mayorga K, Madariaga-Mazon A, Medina-Franco JL, Maggiora G (2020) The impact of chemoinformatics on drug discovery in the pharmaceutical industry. Expert Opin Drug Discov 15(3):293-306. https://doi. org/10.1080/17460441.2020.1696307

16. Fenteany G, Gaur P, Sharma G, Pintér L, Kiss E, Haracska L (2020) Robust high-throughput assays to assess discrete steps in ubiquitination and related cascades. BMC Mol Cell Biol 12(21):1-6. https://doi.org/10.1186/s12 860-020-00262-5

17. García-Serradilla M, Risco C, Pacheco B (2019) Drug repurposing for new, efficient, broad spectrum antivirals. Virus Res 264:22-31. https://doi.org/10.1 016/j.virusres.2019.02.011

18. Senanayake SL (2020) Drug repurposing strategies for COVID-19. Future Drug Discov 0(0) fdd-2020-0010. https://doi.org/10.4155/fdd-2020-0010
19. Shah B, Modi P, Sagar SR (2020) In silico studies on therapeutic agents for COVID-19: drug repurposing approach. Life sciences 252:117652. https://doi. org/10.1016/j.lfs.2020.117652

20. Zhou W, Liu Y, Tian D, Wang C, Wang S, Cheng J, Hu M, Fang M, Gao Y (2020) Potential benefits of precise corticosteroids therapy for severe 2019nCoV pneumonia. Signal Transduct Target Ther 5(1):18. https://doi.org/10.1 038/s41392-020-0127-9

21. Bhimraj A, Morgan RL, Shumaker AH, Lavergne V, Baden L, Cheng VC, Edwards KM, Gandhi R, Muller WJ, O'Horo JC, Shoham S, Murad MH, Mustafa RA, Sultan S, Falck-Ytter Y (2020) Infectious Diseases Society of America Guidelines on the treatment and management of patients with COVID-19. Clin Infect Dis 27:ciaa478. https://doi.org/10.1093/cid/ciaa478

22. Yang $X, Y u$ Y, Xu J, Shu H, Xia J, Liu H, Wu Y, Zhang L, Yu Z, Fang M, Yu T, Wang Y, Pan S, Zou X, Yuan S, Shang Y (2020) Clinical course and outcomes of critically ill patients with SARS-CoV-2 pneumonia in Wuhan, China: a single-centered, retrospective, observational study. Lancet Respir Med 8(5): 475-481. https://doi.org/10.1016/S2213-2600(20)30079-5

23. Zhou F, Yu T, Du R, Fan G, Liu Y, Liu Z, Xiang J, Wang Y, Song B, Gu X, Guan L, Wei Y, Li H, Wu X, Xu J, Tu S, Zhang Y, Chen H, Cao B (2020) Clinical course and risk factors for mortality of adult inpatients with COVID-19 in Wuhan, China: a retrospective cohort study. Lancet 395(10229):1054-1062. https://doi.org/10.1016/S0140-6736(20)30566-3

24. Liu J, Zhang S, Dong X, Li Z, Xu Q, Feng H, Cai J, Huang S, Guo J, Zhang L, Chen Y, Zhu W, Du H, Liu Y, Wang T, Chen L, Wen Z, Annane D, Qu J, Chen D (2020) Corticosteroid treatment in severe COVID-19 patients with acute respiratory distress syndrome. J Clin Invest 130(12):6417-6428. https://doi. org/10.1172/JCl140617

25. Dequin PF, Heming N, Meziani F, Plantefève G, Voiriot G, Badié J, François B, Aubron C, Ricard JD, Ehrmann S, Jouan Y, Guillon A, Leclerc M, Coffre C, Bourgoin H, Lengellé C, Caille-Fénérol C, Tavernier E, Zohar S, Giraudeau B, Annane D, Le Gouge A, CAPE COVID Trial Group, the CRICS-TriGGERSep Network (2020) Effect of hydrocortisone on 21-day mortality or respiratory support among critically ill patients with COVID-19: a randomized clinical trial. JAMA 324(13):1298-1306. https://doi.org/10.1001/jama.2020.16761

26. Ramiro S, Mostard RLM, Magro-Checa C, van Dongen CMP, Dormans T, Buijs J, Gronenschild M, de Kruif MD, van Haren EHJ, van Kraaij T, Leers MPG, Peeters R, Wong DR, Landewé RBM (2020) Historically controlled comparison of glucocorticoids with or without tocilizumab versus supportive care only in patients with COVID-19-associated cytokine storm syndrome: results of the CHIC study. Ann Rheum Dis 79(9):1143-1151. https://doi.org/10.1136/annrheumdis-2020-218479

27. Angus DC, Derde L, Al-Beidh F, Annane D, Arabi Y, Beane A et al (2020) Effect of hydrocortisone on mortality and organ support in patients with severe COVID-19: the REMAP-CAP COVID-19 corticosteroid domain randomized clinical trial. JAMA 324(13):1317-1329. https://doi.org/10.1001/ja ma.2020.17022

28. Mason RJ (2020) Pathogenesis of COVID-19 from a cell biology perspective. Eur Respir J 55(4):2000607. https://doi.org/10.1183/13993003.00607-2020

29. Steinberg KP, Hudson LD, Goodman RB, Hough CL, Lanken PN, Hyzy R, Thompson BT, Ancukiewicz M National Heart, Lung, and Blood Institute Acute Respiratory Distress Syndrome (ARDS) Clinical Trials Network (2006) Efficacy and safety of corticosteroids for persistent acute respiratory distress syndrome. N Engl J Med 354(16):1671-1684. https://doi.org/10.1056/NEJMoa 051693

30. Lee N, Allen Chan KC, Hui DS, Ng EK, Wu A, Chiu RW, Wong W, Chan PK, Wong KT, Wong E, Cockram CS, Tam JS, Sung JJ, Lo YM (2004) Effects of early corticosteroid treatment on plasma SARS-associated coronavirus RNA concentrations in adult patients. J Clin Virol 31(4):304-309. https://doi.org/1 0.1016/j.jcv.2004.07.006

31. Wu C, Chen X, Cai Y, Xia J, Zhou X, Xu S, Huang H, Zhang L, Zhou X, Du C, Zhang Y, Song J, Wang S, Chao Y, Yang Z, Xu J, Zhou X, Chen D, Xiong W, Xu L, Zhou F, Jiang J, Bai C, Zheng J, Song Y (2020) Risk factors associated with acute respiratory distress syndrome and death in patients with coronavirus disease 2019 pneumonia in Wuhan, China. JAMA Intern Med 180(7):934-943. https://doi.org/10.1001/jamainternmed.2020.0994

32. Shang $L$, Zhao J, Hu Y, Du R, Cao B (2020) On the use of corticosteroids for 2019-nCoV pneumonia. Lancet 395(10225):683-684. https://doi.org/10.1016/ S0140-6736(20)30361-5

33. Khilnani GC, Hadda V (2011) Corticosteroids and ARDS: a review of treatment and prevention evidence. Lung India 28(2):114. https://doi.org/1 0.4103/0970-2113.80324 
34. RECOVERY Collaborative Group, Horby P, Lim WS, Emberson JR, Mafham M, Bell JL, Linsell L, Staplin N, Brightling C, Ustianowski A, Elmahi E, Prudon B, Green C, Felton T, Chadwick D, Rege K, Fegan C, Chappell LC, Faust SN, Jaki T, Jeffery K, Montgomery A, Rowan K, Juszczak E, Baillie JK, Haynes R, Landray MJ (2020) (2020) Dexamethasone in hospitalized patients with covid-19 - preliminary report. N Engl J Med NEJMoa2021436. https://doi. org/10.1056/NEJMoa2021436

35. El Hadidi S, Rosano G, Tamargo J, Agewall S, Drexel H, Kaski JC, Niessner A, Lewis BS, Coats AJS (2020) Potentially inappropriate Prescriptions in Heart Failure with Reduced Ejection Fraction (PIP-HFrEF). Eur Heart J Cardiovasc Pharmacother:pvaa108. https://doi.org/10.1093/ehjcvp/pvaa108

\section{Publisher's Note}

Springer Nature remains neutral with regard to jurisdictional claims in published maps and institutional affiliations.

\section{Submit your manuscript to a SpringerOpen ${ }^{\circ}$ journal and benefit from:}

- Convenient online submission

- Rigorous peer review

- Open access: articles freely available online

High visibility within the field

- Retaining the copyright to your article

Submit your next manuscript at $\boldsymbol{\nabla}$ springeropen.com 